\title{
Distribution of injuries among patients of Department of Trauma and Emergency Medicine of Independent Public Teaching Hospital No. 1 in 2011
}

\begin{abstract}
Introduction. Injuries are an extremely important public health problem, in both developed and developing countries. Various traumas contribute to some one third of deaths of the entire human population followed by cardiovascular diseases and cancer only. Understanding the epidemiology of trauma might improve hospitals' ability to provide proper diagnoses and treatment.

Aim. The aim of this study was to provide epidemiological characteristics of injuries in the analyzed group of patients.

Material and methods. A retrospective analysis of patients admitted in state of emergency and hospitalized in the Department of Trauma Surgery and Emergency Medicine at the Medical University of Lublin due to injuries that occurred from January $1^{\text {st }}$ 2011 till December $31^{\text {st }} 2011$ was conducted. Mechanism of injury, mortality rate, age and gender correlations were studied.

Results. Some 485 patients were looked at in the study. The mortality rate reached $4.52 \%$ and was highest among males. Falling was the leading cause of injuries among patients

Discussion. The results of this study correspond with the observations of other authors concerning the age distribution. Mortality rates noticed in this study were lower than in other studies.

Conclusions. Trauma in elderly patients is a growing problem in trauma centers. Moreover, in the analyzed material incidence of blunt and non-transport related injuries increased.
\end{abstract}

Keywords: injury, epidemiology, blunt injury, penetrating injury.

DOI: $10.1515 /$ pjph-2016-0008

\section{INTRODUCTION}

Injuries remain a profound problem of public life not only because of their medical impact but also because they are often considered as an important economical and social factor. Injuries make up for one third of deaths of the entire human population, followed only by cardiovascular diseases and cancer. Moreover, statistics show that injury-related mortality rates are on the rise [1]. They are, however, the leading cause of death among patients up to the age of 44, thus generating huge financial losses not only because of work absences or treatment costs but also because they might cause various disabilities [2]. Years of potential life lost (YPLL) estimate due to injuries is definitely the highest when compared to all other causes of deaths and disabilities. Special diagnostic and therapeutic difficulties occur in case of severe, multiple and multiorgan injuries. This is due to the fact that this type of injuries causes numerous pathophysiological and metabolic effects and elicit a general response from the body.

\section{AIM}

The aim of this study was to provide epidemiological characteristics of injuries in the analyzed group of patients.

\section{MATERIAL AND METHODS}

The authors of this study performed a retrospective analysis on some 485 patients admitted in a state of emergency and hospitalized in the Department of Trauma Surgery and Emergency Medicine at the Medical University of Lublin. The injuries occurred between January $1^{\text {st }} 2011$ and December $31^{\text {st }} 2011$. The main criteria for including the patients in a particular group were the period between the time of injury and moment of administration to the hospital emergency department being not longer than 12 hours. The authors looked at adult patients only. The researched group included patients whose injuries were classified using ICD-10 and were ranged S00-T19, while the mechanism of injury also classified using ICD-10 was ranged V01-Y98.

The database used for analysis was created using the medical records provided by Emergency Medicine Services and the following departments: Hospital Emergency Department, Department of Trauma Surgery and Emergency Medicine, Department of Anesthesiology and Intensive Care of Independent Public Teaching Hospital No. 1 in Lublin.

Following the patients' discharge, a retrospective analysis of individual medical documentation was performed. The injuries were described using S-T codes (according to the ICD-10

\footnotetext{
${ }^{1}$ Chair and Department of Trauma Surgery and Emergency Medicine, Medical University of Lublin, Poland

${ }^{2}$ Department of Orthopaedic Surgery, Independent Public District Hospital in Leczna, Poland

${ }^{3}$ Department of Internal Medicine, Medical University of Lublin, Poland
} 
classification), starting from isolated injuries and ending with multiple injuries coded to fourth disease inclusive, as well as mechanism of the injury from the range V-Y (according to the ICD-10 classification). In addition, the injuries were classified as either blunt or penetrating and the fact whether the patient survived or not was also noted. Penetrating injury was considered as an injury penetrating into the body's cavities. Blunt injury was considered as an injury not affecting the skin continuity. The ICD-10 system was used to distinguish between seven parts of body (head and neck, chest, abdomen, spine and pelvis, upper limb, lower limb, internal injuries and other) and a group where other injuries were placed.

\section{RESULTS}

In 2011, some 485 patients were hospitalized due to injuries at the Department of Trauma Surgery and Emergency Medicine. Out of the study group, some 22 patients died during their hospital stay, which meant a mortality rate of $4.52 \%$ in the group. Male patients were twice more likely to die.

TABLE 1. Comparison of gender proportion in the analyzed group of injured patients considering mortality in individual groups.

\begin{tabular}{lccc}
\hline \hline & Females & Males & Study Group \\
\hline Number of people & 200 & 286 & 486 \\
\hline Percentage of the study group & $41.15 \%$ & $58.85 \%$ & $100 \%$ \\
\hline Number of deaths & 7 & 15 & 22 \\
\hline $\begin{array}{l}\text { Percentage of deaths } \\
\text { in the study group }\end{array}$ & $1.44 \%$ & $3.08 \%$ & $100 \%$ \\
\hline
\end{tabular}

Patients aged 70 and more were the biggest age group (some $30.7 \%$ of the entire study group.) In addition, those aged between 31 and 50 consituted slightly smaller groups (27.2\%), while those aged 51-70 made $26.8 \%$. The smallest group of trauma patients were adolescents and adults under 30 years of age.

It is worth noticing that in case of patients under 30, as well as those of $31-50$ years of age, the percentage of females was respectively $2.7 \%$ and $6.8 \%$ (17.6\% and $25 \%$ of age group) whereas in the same age groups the percentage of males was $12.6 \%$ and $20.4 \%$ ( $82.4 \%$ and $75.0 \%$ of age group). Hence, it is clear that in the age group of patients under 30 , men were injured 4 times more than females $(17.6 \%$ vs $82.4 \%, \mathrm{p}<0.0001)$ whereas in the age group 31-50 there were more than 3 times more males compared to females ( $25 \%$ vs $75 \%, p<0.0001)$. In the age group of 51-70, the patients' gender distribution was similar.

As opposed to age groups below 30 and 31-50, the frequency of injuries in patients over 70 was affected by gender and age. In this age group, women were more often injured. Their percentage in the group was $21.9 \%$ ( $71.1 \%$ of the research group) which compared to the percentage of men $8.9 \%(28.9 \%$ of the research group) shows that they got injured more than twice compared to men. Patients aged between 18 and 70 accounted for $69.3 \%$ of the entire research group, which was dominated by men $-85 \%$. Patients above the age of 70 accounted for $30.7 \%$ of the research group, with women constituting some $71.1 \%$.

In the group of patients that were looked at, those who suffered from a blunt trauma constituted a majority. The proportion was as follows: 442 cases of blunt injuries and 43 penetrating injuries. Males were the dominant gender group in case of penetrating injuries - they were 5 times more likely to suffer from these injuries. Penetrating injuries affected $12.6 \%$ of males from the entire study population, compared to $3.6 \%$ of females from the entire study population.

Blunt injuries constitute the majority of injuries in the study group and women are more likely to experience them. As much as $96.5 \%$ of females from the entire study suffered from blunt trauma, while in case of males it was $87.4 \%$. Practice shows that males are more likely to be victims of sharp objects, as well as fights involving use of a knife or mechanical tools such as chainsaws or circular saws - causing penetrating injuries. Females more frequently suffered from blunt traumas, especially from falls.

When looking at the distribution of blunt and penetrating injuries, some statistical significance was found for two age groups, namely patients up to the age of 30 those above 70 years of age. This significance was found in case of both penetrating and blunt injuries.

In the group of patients up to the age of 30 as many as $14.9 \%$ of them suffered from a penetrating injury. This shows that young people are most likely to suffer from penetrating injuries. The opposite situation can be observed among the oldest patients - over 70, where penetrating injuries accounted for $4 \%$ only. In the group with blunt injuries, the situation is completely different. In this age group the percentage of patients involved is highest after the age of 70 and is $96 \%$, whereas it is low in the youngest age group up to the age of 30 , showing a $85.5 \%$ rate of incidence.

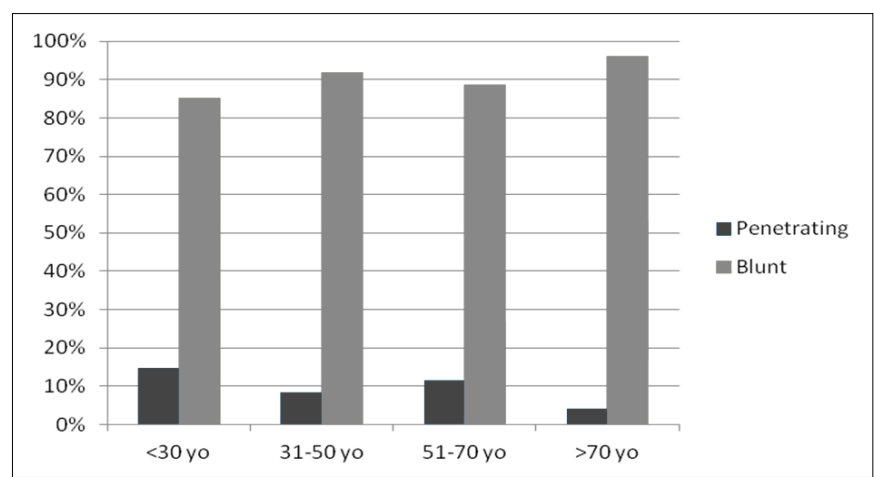

FIGURE 1. Characteristics of types of injuries in different age groups.

In patients of 31-50 and 51-70 age groups, no statistically significant dependence was found.

When considering the total number of patients and their percentage in the entire study group instead of the percentage of a given age group, it turns out that the largest group of patients who suffered from a penetrating injury, were patients in the age group $51-70$ as there were 15 of them, which is $3.1 \%$ of the total. The smallest group suffering from penetrating injuries were patients older than $70-6$ patients, which means $1.2 \%$ of the total. A group of patients older than 70 accounted for an absolute majority of the entire study group with blunt trauma - 143 patients representing $29.5 \%$ of the total and a group of patients under the age of 30 was the smallest one accounting for 63 patients, representing $13 \%$ of total.

In addition to the type of injury, patients were qualified into different groups in terms of mechanism of injury, according to ICD-10 classification. A division of patients from the study group was particularly considered as to whether the injury took place during transportation or not.

Patients' sex had no influence whatsoever over the frequency of traffic-related accidents. 
There were significantly more patients suffering from nontransportation related injuries (419 patients making $86.4 \%$ of the total) than those affected by road accidents (66 patients $13.6 \%$ of the total). The largest group of patients were those who suffered from a non-transportation injury in the age group above 70 . They accounted for $94 \%$ in their age group and for $28.9 \%$ of the whole study group, giving a number of 140 patients. This age group, as shown in the data, were least likely to suffer from traffic-related injuries making $6 \%$ of the studied age group and only $1.9 \%$ of total -9 patients.

The smallest group among non-transportation related injuries consisted of youngest patients, meaning those younger than 30 , making $11.8 \%$ of total and only $77 \%$ in their age group. Those patients were a dominant group among those suffering from traffic-related accidents, compared to other corresponding age groups. In this case they represented up to $23 \%$ of their age group (for comparison this percentage above the age of 70 was $6 \%$ ).

Overall, as the data presented in Figure 2 below shows, traffic-related injuries were most likely to happen to patients under 50 . No statistically significant correlation was found for the age group 51-70 and in the age group above 70 years old, most casualties suffered from accidents not related to traffic.

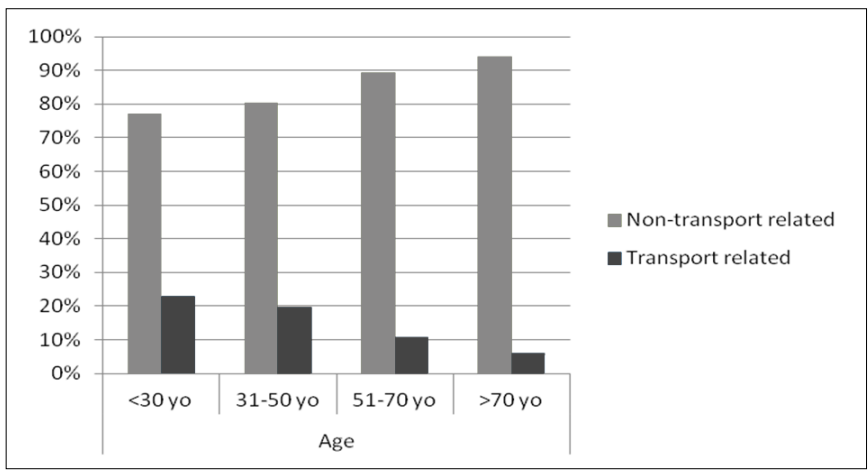

FIGURE 2. Causes of injuries in various age groups.

As it can be clearly seen in the analysis, elderly patients are more likely to suffer from injuries related to falls. This might be associated with their age and related disaeses, whereas younger patients more often suffer from transport-related injuries as a vehicle user, heavy vehicle user, pedestrian, cyclist or biker. As it is shown in Figure 2, the older the patient the higher the incidence of non-transport related injuries and less of transport related injuries.

Statistical calculation was also performed searching for relations among studied patients between blunt, penetrating injuries and mechanisms of injury transport and non-transport related problems. No statistical relation had been found and statistical distribution of these patients presents as in random sampling.

Out of the 485 participants of the study, some 22 patients died. The authors attempted at finding a statistical relationship between gender and whether the patient survived or died but no relation was found.

The relationship between one's age and chances of survival was also looked upon by the authors. Such a correlation was found for patients aged 70 or above. Among the members of this group, deaths occurred significantly more often than in other age groups. Considering the statistically significant relation between blunt, penetrating injuries and survival or death of patients it was shown that it did not exist. The cause of an injury (whether it was traffic or non-traffic-related) had no influence over it either. Deaths in both groups occurred frequently.

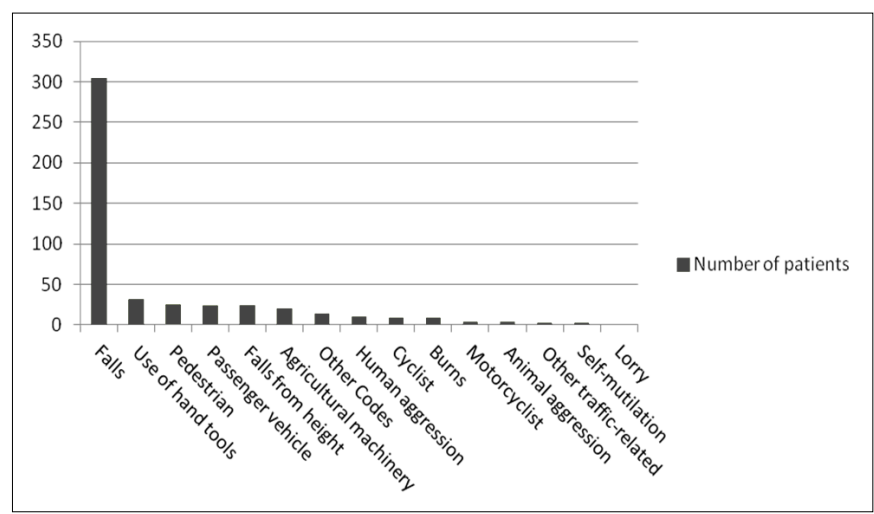

FIGURE 3. Characteristics of number of patients who have suffered injury as a result of various mechanisms of injury.

Figure 3 illustrates the size of the groups of patients who suffered from different types of injury. It has been observed that in the studied group of patients falls were most frequent. Some 350 of them were recorded in the entire group, making the number larger than the total of the rest of the injuries.

Regarding the parts of the body that were most likely to suffer from injuries, it appeared that most injuries happened to the lower limb with a group of 251 patients. Upper limb was in second place in this classification with a number of 104 patients, then head and neck with 61 patients. Other areas of the body that suffered an injury were groups of patients from 10 to 26 . As it can be seen in figure 4 , limb related injuries accounted for $73.2 \%$ of the study group.

\section{DISCUSSION}

The study shows that the most common pattern of injury among patients of Independent Public Teaching Hospital No. 1 were blunt injuries. Moreover, patients were most likely to suffer from injuries resulting from simple falls. Those results correspond with the findings by Swiss authors who conducted a 5-year review from a Swiss trauma center. In that case, blunt injuries were the most common cause of admission to a trauma center [3]. However, as far as the patients' age upon admission is considered, there was a discrepancy in results. In our study, most patients were women over 70 , while the authors mentioned above noticed that men in their early 40 s were the greatest number of patients. On the other hand, our findings are similar to what a Canadian study (a study that retrospectively reviewed hospital admissions between 2000 and 2003) reavealed [4]. Elderly patients covered $30 \%$ of patients admitted to trauma center. In this study authors showed that elderly patients account for more than $50 \%$ of trauma-related deaths, which is similar to our findings. However, elderly patients suffer from many comorbidities, and after being subjected to trauma, their very limited physiological reserves may predispose them to death from a less severe trauma. A study published in 2013 corresponds with our results and shows an increase in trauma affecting the elderly [5]. This shows that trauma centers are increasingly more likely to deal with patients suffering from many comorbidities, and therefore may require an individualized approach. Moreover, elderly patients are most susceptible to injuries which take place in home environment [6]. 
In 2006, American authors published an article in which they looked at mortality rates in 52 hospitals, out of which one was level one trauma centre, and 51 were non-trauma hospitals, located in 14 states. Researchers found that mortality rate is higher in non-trauma hospitals than in trauma centers, respectively $9.5 \%$ vs $7.6 \%$ [7]. In our study, the morality rate was $4.52 \%$. The discrepancy of results seems to be due to methodology of the study. In our study we have evaluated all patients admitted to hospital with trauma, whereas in mentioned study only patients affected by either moderate or severe trauma were included. Nevertheless, these results show that a multidisciplinary approach to patients with injuries in trauma hospitals enhances survival rates.

A significant difference was found when considering trafficrelated trauma, which in our study represented $13.6 \%$, whereas other authors report percentage ranging from $33 \%$ to $40 \%$ [3].

\section{CONCLUSIONS}

1. The most common cause of injuries in the study group of patients were falls from standing position both in male and female groups, whereas the cause of severe injuries were transport-related accidents.

2. In the analyzed material incidence of blunt and non-transport related injuries increased.

3. The most common area of the body that became injured was the lower limb, then the upper limb and head. Males were injured much more often than females and the percentage of deaths in the male group was more than twice higher compared to female group.

\section{REFERENCES}

1. Heron M. Deaths: Leading Causes for 2012. Natl Vital Stat Rep. 2015;64:1-93

2. Sanchez JI, Paidas CN. Childhood trauma. Now and in the new millennium. Surg Clin North Am. 1999;79:1503-35.

3. Heim C, Bosisio F, Roth A, et al. Is trauma in Switzerland any different? Epidemiology and patterns of injury in major trauma - a 5-year review from a Swiss trauma centre. Swiss Med Wkly. 2014;144:w13958.

4. Gowing R, Jain MK. Injury patterns and outcomes associated with elderly trauma victims in Kingston, Ontario. Can J Surg. 2007;50:437-44.

5. Ciesla, DJ, Pracht EE, Tepas JJ 3rd, et al. The injured elderly: a rising tide. Surgery. 2013;154:291-8.

6. Runyan, CW, Perkis D, Marshall SW, et al. Unintentional injuries in the home in the United States Part II: morbidity. Am J Prev Med. 2005;28:807.

7. MacKenzie, EJ, Frederick P, Rivera MD, et al. A national evaluation of the effect of trauma-center care on mortality. N Engl J Med. 2006;354:366-78.

Corresponding author

MD PhD Mariusz Jojczuk

Chair and Department of Trauma Surgery and Emergency Medicine, Medical University of Lublin

16 Staszica Str., 20-081 Lublin

tel +48 0815321854

E-mail: mariuszjojczuk@o2.pl 